\title{
Automatic detection of welding defects using the convolutional neural network
}

\author{
Roman Sizyakin ${ }^{\mathrm{a}}$, Viacheslav Voronin ${ }^{\mathrm{a}, \mathrm{b}}$, Nikolay Gapon ${ }^{\mathrm{a}}$, Aleksandr Zelensky ${ }^{\mathrm{b}}$, and \\ Aleksandra Pižurica ${ }^{\mathrm{c}}$ \\ aLab. "Mathematical methods of image processing and intelligent computer vision systems", \\ Don State Technical University, Rostov-on-Don, Russia \\ bMoscow State University of Technology "STANKIN", Moscow, Russia \\ ${ }^{\mathrm{c}}$ Department Telecommunications and Information Processing, TELIN-GAIM, Ghent \\ University, Ghent, Belgium
}

\begin{abstract}
Quality control of welded joints is an important step before commissioning of various types of metal structures. The main obstacles to the commissioning of such facilities are the areas where the welded joint deviates from acceptable defective standards. The defects of welded joints include non-welded, foreign inclusions, cracks, pores, etc. The article describes an approach to the detection of the main types of defects of welded joints using a combination of convolutional neural networks and support vector machine methods. Convolutional neural networks are used for primary classification. The support vector machine is used to accurately define defect boundaries. As a preprocessing in our work, we use the methods of morphological filtration. A series of experiments confirms the high efficiency of the proposed method in comparison with pure CNN method for detecting defects.
\end{abstract}

Keywords: Welding defect detection, convolutional neural network (CNN), support vector machine (SVM), morphological filtering, histogram equalization

\section{INTRODUCTION}

Analysis of the quality of welded joints is a mandatory procedure before commissioning of such facilities as gas pipelines, heating pipelines and other types of metal structures. This analysis is necessary for the detection and localization of various types of defects on the weld. Defects of welded joints significantly reduce the overall durability of the entire structure, ignoring which can subsequently lead to significant human-made consequences. There are two main types of analysis of the quality of welded joints: destructive and non-destructive. Currently, non-destructive testing methods are used to a greater extent. At the moment, there are a large number of non-destructive testing methods aimed at detecting defects in welded joints. The most informative and effective are considered to be radiographic methods of non-destructive testing.

At the initial stage, sites with suspected defects are analyzed using x-rays. On the opposite side of the place with the alleged defects, a special film or plate is installed, which fixes the amount of energy passed through the material under study. After that, the film is scanned and converted into digital form. At the final stage, the digitized image is recognized by a flaw detection engineer. This process can take a significant amount of time. For example, it may take a specialist 10 to 20 minutes to recognize and archive a single ring weld. In the case of, for example, a multi-kilometer section of the pipeline, this process can take more than one hour. Also, it should be noted that due to fatigue, negligence, lack of training or a combination of other human factors, the flaw detector engineer can make involuntary mistakes that can lead to various production consequences.

Significantly simplify, and in some cases completely solve the problem of visual analysis of the digitized images, can use the latest achievements in the field of digital signal processing and computer vision. Modern studies show that computer vision systems can achieve results in the analysis and classification of images close to the results obtained in the visual analysis of these images by a human. This statement is confirmed by research in the field of artificial intelligence based on the use of neural network technologies. ${ }^{1}$ 


\section{RELATED WORK}

Detection of weld defects based on the analysis of local textural image features was studied in work. ${ }^{2}$ A set of Gabor filters ${ }^{3}$ and Haralick features ${ }^{4}$ were used to calculate textural features of the image. In total, 14 features of Haralick used, among which: contrast, correlation, entropy, dispersion, etc. The nearest neighbor method was used as a classifier. ${ }^{5}$

In work, ${ }^{6}$ the authors used spatial image segmentation to detect defects in welded joints. The method consists of three main steps: filtration, correction of uneven lighting, segmentation. To reduce the noise component, a local adaptive Wiener filter ${ }^{7,8}$ and an adaptive digital filter with fuzzy $\operatorname{logic}^{9}$ were used. For the correction of lighting used morphological image filtration, as well as processing in the frequency domain, using a fast discrete Fourier transform ${ }^{10}$ were applied. At the final stage, the image was segmented using the "triangle" method, ${ }^{11}$ to detect defect.

A defect detection method based on multistage processing of the image, with the subsequent classification using a neural network was proposed in work. ${ }^{12}$ At the initial stage, the image was pre-processed by reducing the noise component using the Wiener filter, improving the contrast of the image using an adaptive histogram enhancing (AHE). ${ }^{13} \mathrm{~A}$ discrete wavelet transform was employed to reduce the area for further processing. After all the transformations, the image pixels were classified using a fully connected neural network. Skewness and kurtosis coefficients and Mel Frequency Cepstral Coefficients (MFCCs) were used as descriptors. ${ }^{14,15}$

The method of defect detection proposed in work ${ }^{16}$ is based on the calculation of a set of texture descriptors, followed by classification based on a neural network. In total, twenty-two texture features were used, including energy, correlation, contrast, and other, based on the works of Clausi, ${ }^{17}$ Soh $^{18}$ and Haralick. ${ }^{4}$

In work, ${ }^{19}$ the authors employed a deep neural network architecture to detect defects in welded joints. To reduce the computational complexity, the threshold method of $\mathrm{Otsu}^{20}$ was applied. This method allows dividing the image into two components: the background (which is not used at the stage of classification) and the foreground (all pixels of this area are subject to classification).

The main disadvantage of the above works is that, to detect cracks, it is necessary to use various texture descriptors, which in turn lead to an increase in computational costs. The closest to our work is the work. ${ }^{19} \mathrm{~A}$ limitation of that work is an excessive increase in the boundaries of defects.

In this paper, we investigate a new method for detecting defects in welded joints. The proposed method is based on deep learning, followed by accurate detection of defect boundaries using the support vector machine.

\section{MODEL OF WELDING DEFECTS}

Defects of welded connections represent any deviations from the parameters of connections set by normative documents which were formed owing to violation of the technological process of welding. Such violations may be errors associated with the choice of welding technology, violation of the welding process, welding materials of poor quality, etc. There are six main types of defects in welded joints: cracks, cavities and pores, solid inclusions, non-melting and non-welding, violation of the weld shape, and other defects (Figure 1). In our case, the mathematical model of the image containing the defect can be represented as follows:

$$
Y_{i, j}=\left(1-d_{i, j}\right) \cdot S_{i, j}+d_{i, j} \cdot c_{i, j}
$$

where $Y_{i, j}$ - image containing various defects, $i=\overline{1, I}$ and $j=\overline{1, J}$ spatial coordinate, where $I$ and $J$ - height and width of the image in pixels, $S_{i, j}$ - undamaged image, $d_{i, j} \in\{0,1\}$ a binary mask of defects, which shows exactly what area in the image was damaged, $c_{i, j}$ - mask, which contains the brightness values of defects.

\subsection{Cracks}

Cracks in welds represent a break in the weld. Defects of this type can be divided into several types longitudinal, transverse, radial, separate, branched, etc. (Figure 1(a)). The reasons for the formation of this type of defects include the high cooling rate of the welded joint, exceeding the maximum load, design errors, the use of lowquality source material. Cracks are one of the most dangerous and unacceptable types of defects. 


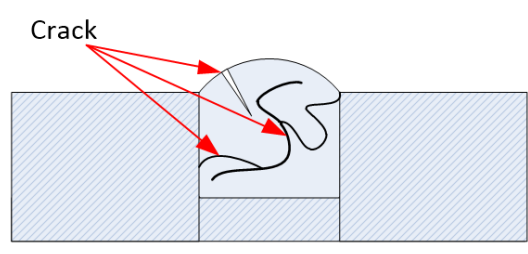

a)

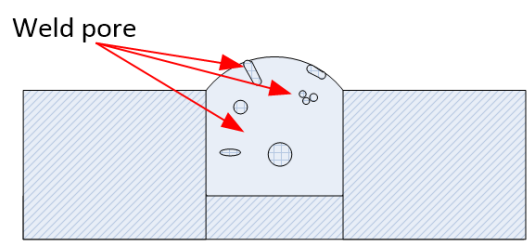

b)

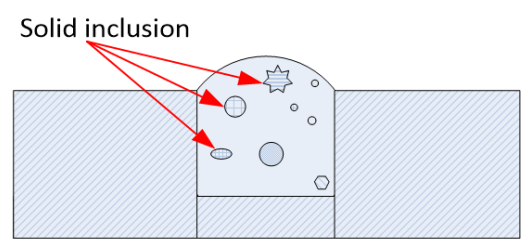

c)

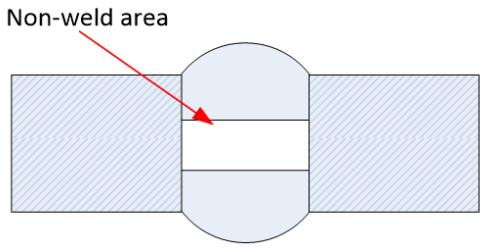

d)

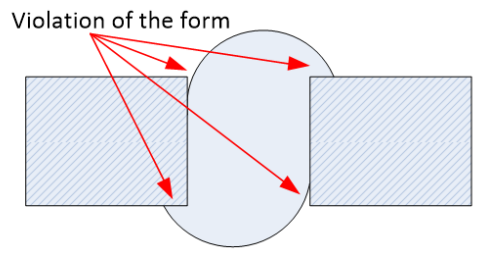

e)

Figure 1. Illustration of different types of weld defects: a) illustration crack, b) illustration weld pore, c) illustration solid inclusion, d) illustration non-weld area, e) illustration violation of the form

\subsection{Weld pore}

The appearance of this type of defect is due to the delay or gas outlet in the welded joint. Pores are divided into several basic types single, accumulation or series of pores, tubular pore, surface pore, crater, shrinkage sink, etc. (Figure 1(b)). The reason for the appearance of pores is caused by the use of poor quality material, poor or insufficient surface treatment of welded materials, short duration of the weld pool, etc. Pores with a small amount do not belong to unacceptable defects of welded joints. However, their presence may be a signal to recheck the welding technology.

\subsection{Solid inclusion}

The appearance of this type of defect is due to the appearance of a foreign substance in the welded joint. Solid inclusions are divided into slag, flux, oxide, and metal (Figure 1(c)). In the process of melting and subsequent mixing of electrode metal, flux, atmospheric gas, and metal surfaces to be welded, particles that do not have time to float to the surface of the weld pool, form these inclusions. Like pores, small solid inclusions do not pose a danger but can lead to a decrease in the wear resistance of the entire structure.

\subsection{Non-weld area}

Non-melting is the absence of a joint between the surface to be welded and the weld metal or the absence of a joint between the weld beads. The failure of the molten metal to reach the root of the weld (Figure $1(\mathrm{~d})$ ) is referred to as non-welding. The main causes of non-melting include an insufficient melting temperature in local places of the weld, improper preparation of welded areas, insufficient welding current for melting metals, etc. The main reasons for non-welding include; the formation of a layer of liquid metal, which prevents the penetration of deeper layers, insufficient cleaning of welding sites, as well as insufficient amperage for the necessary melting of metals. These types of defects, as well as cracks, are dangerous, and ignoring them can lead to increased technological risks.

\subsection{Violation of the welding form}

This type of defect is the deviation of the geometry of the welded joint from the established standard. This type of defects includes various undercuts of the welded joint, excessive weld influx, the formation of small grooves, various kinds of the roughness of the weld surface, etc. (Figure 1(e)). The reasons for the appearance of undercuts are due to the use of an irregularly shaped electrode, improper electrode movement, excessive voltage, or current of the welding arc. The reasons for the influx are due to excessive welding speed, the incorrect inclination of the welded parts or electrode, incorrect voltage, or currently selected for welding. The reasons for the appearance of an uneven surface of the welded joint should include an incorrect choice of welded electrodes, current, and voltage, poor preparation of parts for welding. 


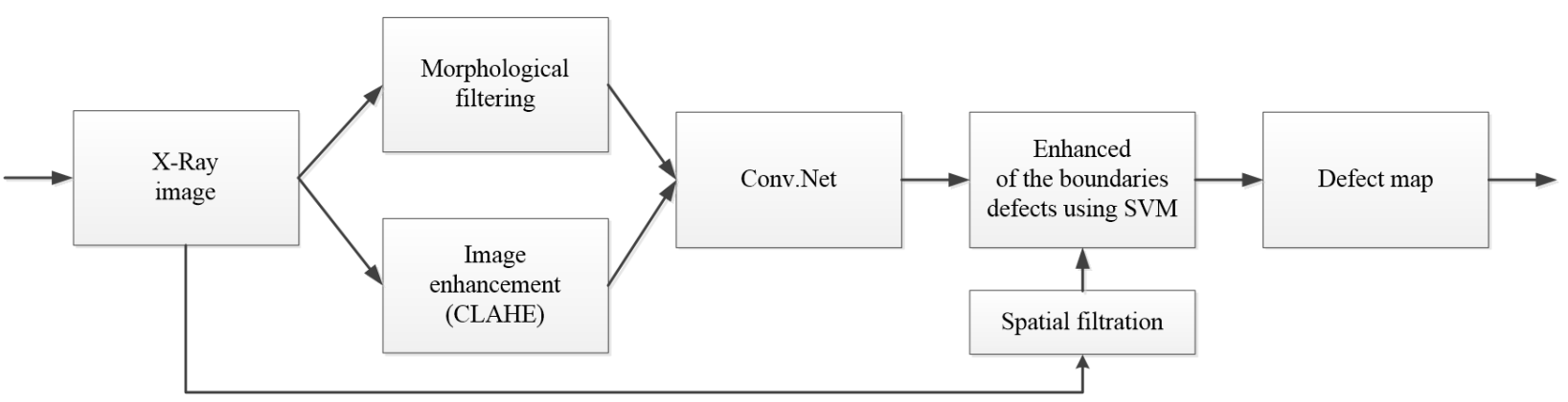

Figure 2. General scheme of the proposed method

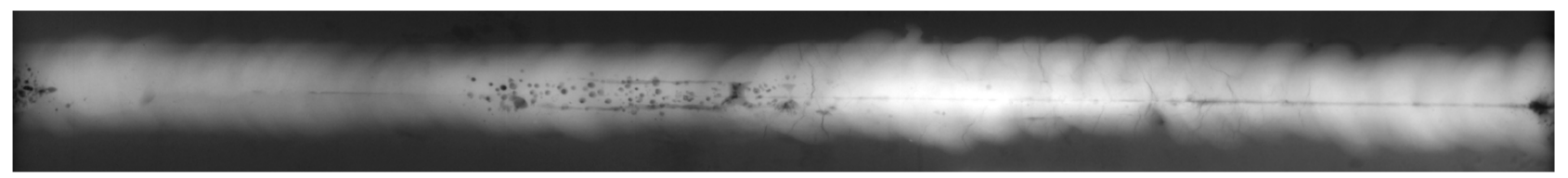

a)

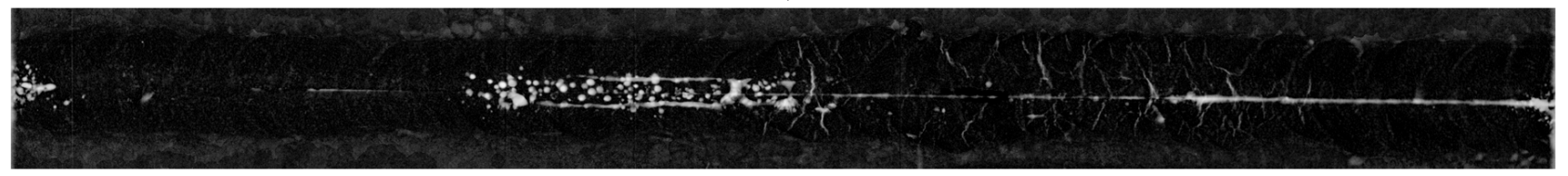

b)

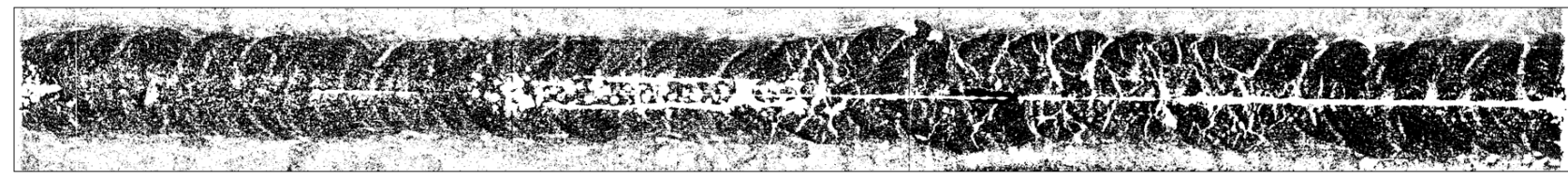

c)

Figure 3. Preliminary localization of defects using morphological filtration: a) source image, b) filtered image, ) thresholded image

\subsection{Other types of defects}

Other defects include defects that are not included in the description above. These may be metal splashes, surface scratches, excessive metal reduction, random arcs, etc. Summarizing a brief description of the main types of defects, it should be noted that different defects have different effects on the overall strength of the welded structure. For example, vapors and solid inclusions in a small amount with a total area of $7 \%$ of the total cross-section of the weld make a slight violation of the strength of the metal structure, which in some cases can be neglected. The defects, which in some cases can be neglected and not eliminated, include excessive convexity, since it does not reduce the overall strength of the structure, but can lead to a decrease in the strength of the structure under vibration load. The defects that are unacceptable include all kinds of cracks, non-proofing, burns, craters, oxide films, non-fusion, as well as almost all kinds of undercuts and surges.

In our work, we will focus on detection on detection of four types of defects: cracks, non-welded, pores, and solid inclusions.

\section{PROPOSED METHOD}

The proposed method consists of three main steps: preliminary localization of the defects using morphological filtering, defect classification using a convolutional neural network, an accurate determination of the boundaries of the defects, using a support vector machine. The general scheme of the proposed method is shown in Figure 2 . 


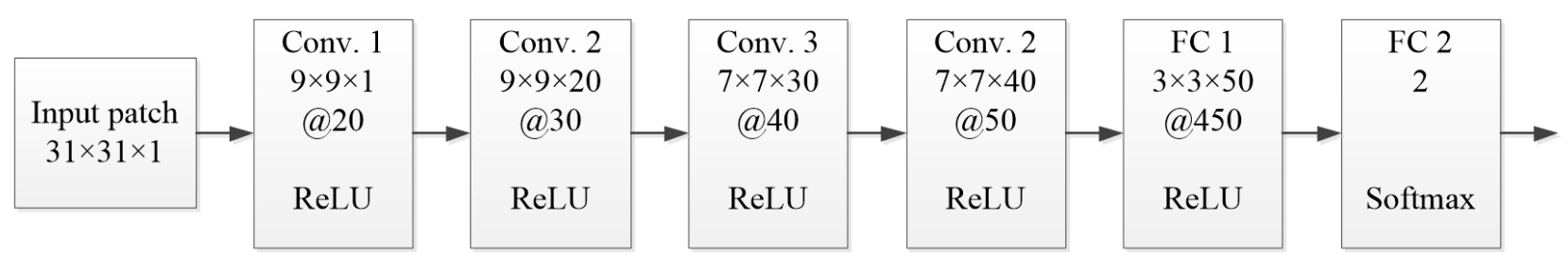

Figure 4. General scheme of the proposed method

\subsection{Morphological filtering}

Morphological image filtering is one of the popular techniques used for the preliminary localization of defects in the image. It allows to reduce the computational cost of the entire algorithm and in some cases, reduce the probability of false alarms. ${ }^{21}$ In our work, we use the "top" and "bottom hat" transform. "Top" and "bottom hat' transform based on four operations of binary mathematical morphology (opening, closing, erosion, and dilation):

$$
\begin{gathered}
\operatorname{BottomHat}\left(Y_{i, j}, B\right)=\left(\left(Y_{i, j} \oplus B\right) \ominus B\right)-Y_{i, j}, \\
\operatorname{TopHat}\left(Y_{i, j}, B\right)=Y_{i, j}-\left(\left(Y_{i, j} \ominus B\right) \oplus B\right)
\end{gathered}
$$

where $Y_{i, j}$ is a grayscale image, $B$ is a structural element, $\left(\left(Y_{i, j} \oplus B\right) \ominus B\right)$ - morphological operation "Close", $\left(\left(Y_{i, j} \ominus B\right) \oplus B\right)$ - morphological operation "Open", $\ominus$ and $\oplus$ corresponds to "Erode" and "Dilate" operations.

The size of the structural element $B$ in our work is set by $20 \times 20$ pixels. To binarize obtained result we use threshold value equal to 0.01. An example of preliminary localization of defects is shown in Figure 3.

\subsection{Classification of defects using a convolutional neural network}

After preliminary localization of defects, each pixel marked with a unit is classified using a convolutional neural network. Convolutional neural networks have several advantages in comparison with traditional methods of machine learning: no need to use different texture descriptors, high accuracy of data classification, the ability to effectively use graphics accelerators to speed up the training and classification processes. ${ }^{22}$ The architecture of the proposed convolutional neural network is illustrated in Figure 4.

At the initial stage of neural network training, kernels in convolutional layers are set by random values. Then for each input image maps of features are calculated by convolution of the image and some kernel. In a simplified form, the convolution function can be defined as:

$$
x_{h, v}^{l}=f\left(\sum_{h} \sum_{v} x_{h+m, v+n}^{l-1} \cdot k_{h, v}^{l}+b\right)
$$

where $x_{h, v}^{l}$ feature map (of a layer $l$ ), $k_{h, v}^{l}$ - the convolution kernel, $f$ is the activation function of the hidden layer, $x_{h+m, v+n}^{l-1}$ - map of features of the previous layer and $b$ - biases.

In hidden layers, a rectified linear unit (ReLU) is used as the activation function: ${ }^{22}$

$$
f(x)=\max (0, x)
$$

where $x$ is the input value of the feature vector/map.

As an activation function in the fully connected layer 2, the "softmax" function is used, which is defined by the expression:

$$
y^{\prime}=\frac{e^{z}}{\sum_{r=1}^{n c} e^{z_{r}}}
$$


where $n c$ - number of classes and $z$ is the output value(probability) for the current patch after passing through the convolution neural network. To determine the losses in our work, we use the binary cross-entropy function. This is defined according to the expression:

$$
\operatorname{Loss}\left(y, y^{\prime}\right)=-\frac{1}{N} \sum_{n=1}^{N}\left[y \cdot \log \left(y^{\prime}\right)+(1-y) \cdot \log \left(1-y^{\prime}\right)\right]
$$

where $y^{\prime}$ is the label predicted by our classifier, and $y$ is the ground truth label.

The architecture of the proposed neural network includes four hidden layers and two fully connected layers. The first convolutional layer includes 20 feature maps, the second 30, the third 40, the fourth 50 . The first fully connected layer has a dimension of 450 bins. The momentum is 0.9 . Training it took approximately 60 epochs. For training, the method of optimization Adam proposed in work ${ }^{23}$ is used.

\subsection{Image enhancement and spatial filtration}

To increase classification accuracy Conv.Net, the input X-Ray image is pre-processed using the method of contrast-limited adaptive histogram equalization (CLAHE). ${ }^{24}$ This method is an improvement of the histogram equalization (HE) method, in which histogram equalization occurs for the entire image directly.

The main disadvantage of the method (HE) is the increase in the noise component in areas with close to constant contrast. To correct this disadvantage in the CLAHE method, the histogram equalization is performed in non-intersecting tiles into which the original image is evenly divided. After that, the cumulative distribution function $(\mathrm{CDF})$ histogram is calculated in each tile, based on the selected restrictions. Using CDF values, determine the equalized brightness values for the four neighborhood tiles, for the pixel to which the brightness equalization is applied. The use of bilinear interpolation eliminates artificially induced boundaries.

The spatial filtering block shown in figure 5 is used to extend the number of input modalities by spatially filtering the source x-ray image. In our work used: image obtained by morphological filtering bottom hat, and the size of the structural element 20 pixels, image obtained by equalization the histogram (HE), image obtained by adaptive equalization of the histogram (CLAHE), image obtained by gamma correction and parameter $\mathrm{g}=0.55$. This procedure allows creating an imaginary multimodal source dataset that is used in the block of accurate determination of the boundaries of defects.

\subsection{Accurate determination of the boundaries of the defects}

One of the limitations of convolutional neural networks is their inability to precisely determine the position of the objects of interest in the image. This problem is caused by high volatility of the object of interest inside the patch for classification. For example, this problem is evident in the results in work, ${ }^{19}$ where in addition to the true defects, the detected defect map also contains false positives in the neighborhood of the defect.

To solve this problem, we use the support vector machine (SVM), ${ }^{25}$ to reclassify the boundary pixels. Using SVM allows to more accurately separate pixels belonging to a defect from pixels close to the defect, but belonging to the background. In our work support, vector machine used a linear function with a soft margin of 0.05 to construct the separating hyperplane. The vector passing through 6 imaginary modalities described above is used as the initial data. Approximately 164,000 vectors passing through pixels belonging to defects were used for training, as well as approximately 163,000 vectors passing through areas that do not contain defects.

\section{EXPERIMENTAL RESULTS}

To estimate the effectiveness of the proposed method in our work as the initial data, we use a public database of x-ray images of welded joints GDXray. ${ }^{26}$ For training convolutional neural network(CNN) and support vector machine(SVM) were used labeling x-ray images of a series W0001 (W0001_0003, W0001_0004, W0001_0006, W0001_0008, W0001_0009, and W0001_0010). Images from the same series are used as ground truth labeled images (W0001_0001, W0001_0002, W0001_0005, W0001_0007).

As quantitative metrics are used: 


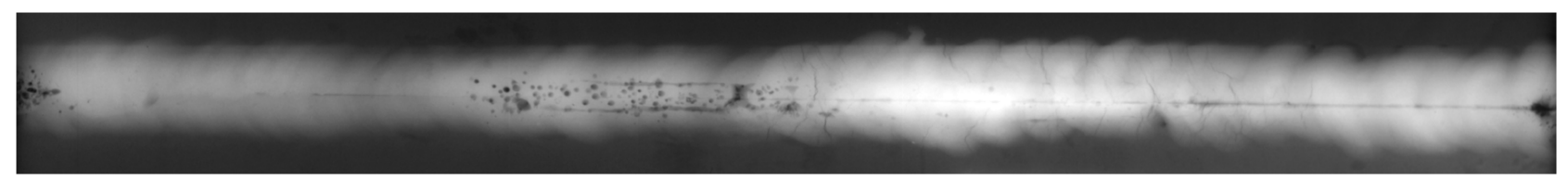

a)

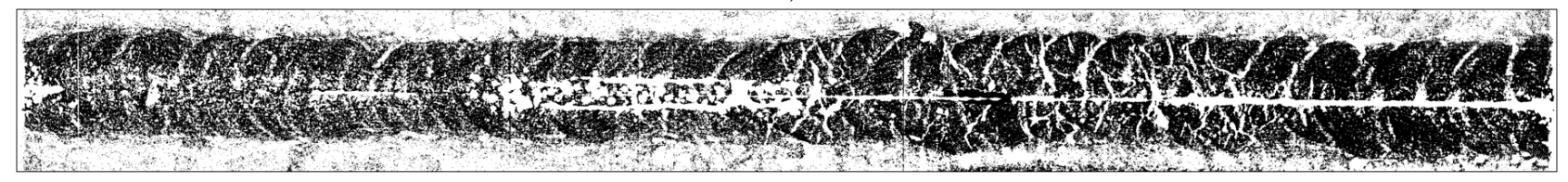

b)

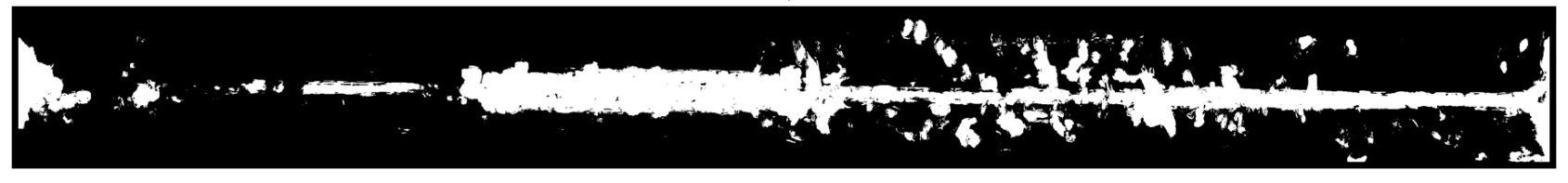

c)

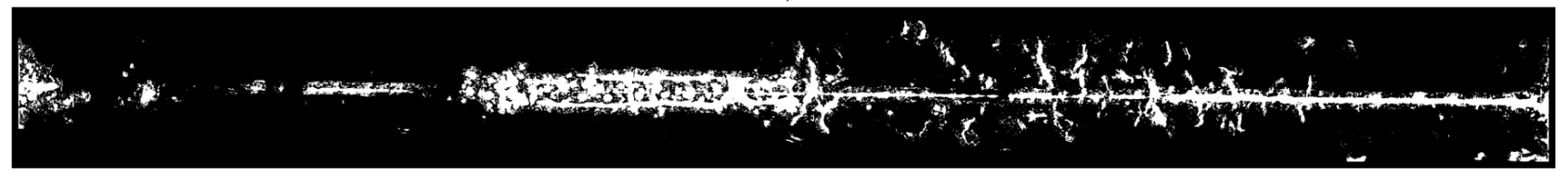

d)

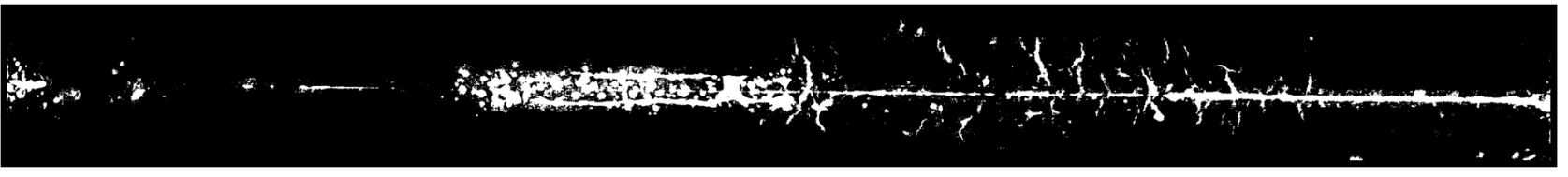

e)

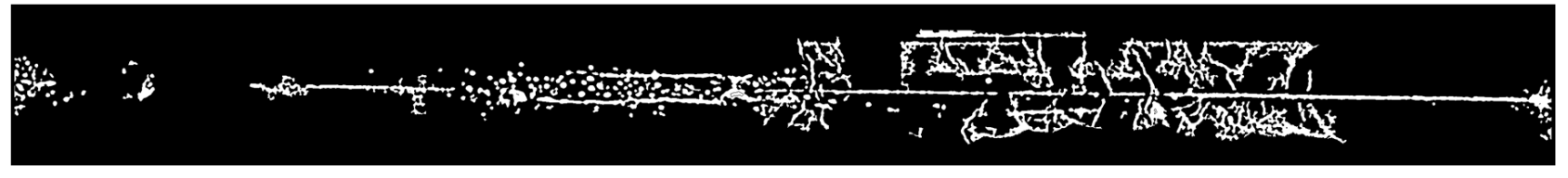

f)

Figure 5. Illustration of the defect detection process for the first test image: a) source image, b) thresholded morphological image, c) Conv.Net, d) Conv.Net + morphological filtering (MF), e) Conv.Net + morphological filtering + enhanced boundaries, f) ground truth defect map

$$
F A=\frac{F P}{\text { AllPix }- \text { DefPix }}, P=\frac{T P}{T P+F P}, R=\frac{T P}{T P+F N}, F 1=\frac{2 P R}{P+R}
$$

where $F A$ - false positives (false alarm), $P$ - is precision, $R$ - is recall, $F 1$ - is F1 measure, AllPix - is total amount of pixels image (equal $I \times J)$ and DefPix - is total amount of defect pixels (equal $\sum_{i=1}^{I} \sum_{j=1}^{J} d_{i, j}$ )

It is important to note that the visual analysis obtained a result when using the only convolutional neural network, without the use of preprocessing in the form of morphological filtering, as well as post-processing in the form of reclassification of boundary pixels, is very close to the result presented in work. ${ }^{19}$ Figure 5 shows an example of defect detection for the first test image W0001_0001. Table 1 summarizes the quantitative metrics for the test images.

Analysis of the results shows the high efficiency of the developed method. The additional use of the support vector machine as a post-processing technique has significantly reduced the probability of false alarms. Thus, 
Table 1. Experimental results for the four test images (MF - is a morphological filtering, EB - is an enhanced boundary)

\begin{tabular}{|c|c|c|c|c|c|}
\hline Test image & Method & Recall & False alarm & Precision & F1-measure \\
\hline \multirow{3}{*}{ W0001_0001 } & Conv.Net. & 0.5882 & 0.1326 & 0.2991 & 0.3966 \\
\cline { 2 - 6 } & MF+Conv.Net & 0.5367 & 0.0554 & 0.4826 & 0.5083 \\
\cline { 2 - 6 } & MF+Conv.Net.+EB & 0.4552 & 0.0278 & 0.6120 & $\mathbf{0 . 5 2 2 1}$ \\
\hline \multirow{3}{*}{ W0001_0002 } & Conv.Net. & 0.8586 & 0.0070 & 0.3296 & 0.4764 \\
\cline { 2 - 6 } & MF+Conv.Net & 0.8451 & 0.0043 & 0.4415 & 0.5800 \\
\cline { 2 - 6 } & MF+Conv.Net.+EB & 0.6921 & 0.0015 & 0.6472 & $\mathbf{0 . 6 6 8 9}$ \\
\hline \multirow{3}{*}{ W0001_0005 } & Conv.Net. & 0.8556 & 0.0118 & 0.4139 & 0.5579 \\
\cline { 2 - 6 } & MF+Conv.Net & 0.8122 & 0.0071 & 0.5288 & 0.6405 \\
\cline { 2 - 6 } & MF+Conv.Net.+EB & 0.7796 & 0.0060 & 0.5577 & $\mathbf{0 . 6 5 0 2}$ \\
\hline \multirow{3}{*}{ W0001_0007 } & Conv.Net. & 0.8615 & 0.0768 & 0.2932 & 0.4375 \\
\cline { 2 - 6 } & MF+Conv.Net & 0.8233 & 0.0526 & 0.3665 & 0.5073 \\
\cline { 2 - 6 } & MF+Conv.Net.+EB & 0.7318 & 0.0223 & 0.5478 & $\mathbf{0 . 6 2 6 6 6}$ \\
\hline
\end{tabular}

the probability of false alarm in most cases for the developed method is lower when compared with a standard convolutional neural network. The main part of false alarms of the convolutional neural network without precise detection of defect boundaries is associated with the high volatility of classified objects inside the patch.

\section{CONCLUSION}

In this paper, we present the main types of defects on welded joints. A new method of detecting various types of defects based on deep learning is also proposed. The main distinguishing feature is the post-processing of the defect map to reduce the probability of false alarms caused by excessive thickening of the defect boundaries. To accomplish this, we use a support vector machine that uses imaginary modality as the input. As a pre-processing, we use morphological filtering, which allows to reduce the computational cost, as well as to reduce the probability of false alarms. The analysis of the experimental results confirmed the high efficiency of the developed method, in comparison with the classification method based on the use of a convolutional neural network alone.

\section{Acknowledgments}

This work was supported by Russian Ministry of Education and Science in accordance to the Government Decree 218 from April 9, 2010 (project number 074-11-2018-013 from May 31, 2018 (03.G25.31.0284)).

\section{REFERENCES}

[1] Russakovsky, O., Deng, J., Su, H., Krause, J., and et al., "Imagenet large scale visual recognition challenge," CoRR abs/1409.0575 (2014).

[2] Mery, D. and Berti, M. A., "Automatic detection of welding defects using texture features," International Symposium on Computerized Tomography and Image Processing for Industrial Applications in Radiology (2003).

[3] Daugman, J. G., "Uncertainty relation for resolution in space, spatial frequency, and orientation optimized by two-dimensional visual cortical filters," Optical Society of America 2(7), 1160-1169 (1985).

[4] Haralick, R., Shanmugam, K., and Dinstein, I., "Textural features for image classification," IEEE Transactions on Systems, Man and Cybernetics 3(6), 610-621 (1973).

[5] Altman, N. S., "An introduction to kernel and nearest-neighbor nonparametric regression," The American Statistician 46 (3), 175-185 (1992). 
[6] Kountchev, R. K., Rubin, S. H., Todorov, V. T., and Kountcheva, R. A., "Automatic detection of welding defects," Int. J. Reasoning-based Intelligent Systems 3(1) (2011).

[7] Lim, J., "Two-dimensional signal and image processing," Prentice Hall, New Jersey (1990).

[8] Gonzalez, R. and Woods, R., "Digital image processing," Prentice Hall, New Jersey (2002).

[9] Kountchev, R., Milanova, M., Todorov, V., and Kountcheva, R., "Adaptive fuzzy filter for the reduction of blocking artifacts in images compressed with idp decomposition and jpeg," WSEAS Transactions on Signal Processing 2(7), 941-948 (2006).

[10] Chu, E. and George, A., "Inside the fft black box: Serial and parallel fast fourier transform algorithms," CRC Press, Boca Raton, FL (2000).

[11] Young, I., Gerbrands, J., and van Vliet, L., "Image processing fundamentals," Delft, The Netherlands (1996).

[12] Saber, S. and Selim, G. I., "Higher-order statistics for automatic weld defect detection," Journal of Software Engineering and Applications 6, 251-258 (2013).

[13] Coltuc, D., Bolon, P., and Chassery, J., "Exact histogram specification," IEEE Transactions on Image Processing 15 (5), 1143-1152 (2006).

[14] Kasban, H., Zahran, O., Arafa, H., El-Kordy, M., Elaraby, S. M. S., and El-Samie, F. E. A., "Welding defect detection from radiographic image using cepstral approach," Nondestructive Testing and Evaluation: An International Journal 44 (2), 226-231 (2011).

[15] Kasban, H., "Applying advanced digital signal processing techniques in industrial radioisotopes applications," Ph.D. Thesis, Faculty of Electronic Engineering, Menofia University, Shibin Al Kawm (2012).

[16] Kumar, J., Anand, R., and Srivastava, S., "Multi - class welding flaws classification using texture feature for radiographic images," International Conference on Advances in Electrical Engineering (ICAEE) (2014).

[17] Clausi, D., "An analysis of co-occurrence texture statistics as a function of grey level quantization," Canadian Journal of remote sensing 28, 45-62 (2002).

[18] Soh, L.-K. and Tsatsoulis, C., "Texture analysis of sar sea ice imagery using gray level co-occurrence matrices," IEEE Transactions on Geoscience and Remote Sensing 37, 780-795 (1999).

[19] Hou, W., Wei, Y., Guo, J., Yi, J., and Changan, Z., "Automatic detection of welding defects using deep neural network," 10th International Conference on Computer and Electrical Engineering, IOP Conf. Series: Journal of Physics: Conf. Series 933 (2018).

[20] Otsu, N., "A threshold selection method from gray-level histograms," IEEE Transactions on Systems, Man, and Cybernetics 9, 62-66 (1979).

[21] Sizyakin, R., Cornelis, B., Meeus, L., Martens, M., Voronin, V., and Pižurica, A., "A deep learning approach to crack detection in panel paintings," Image Processing for Art Investigation (IP4AI) (2018).

[22] Krizhevsky, A., Sutskever, I., and Hinton, G., "Imagenet classification with deep convolutional neural networks," Advances in Neural Information Processing Systems, 1097-1105 (2012).

[23] Kingma, D. and Ba, J., "Adam: A method for stochastic optimization," CoRR (2014).

[24] Zuiderveld, K., "Contrast limited adaptive histograph equalization," Graphic Gems IV. San Diego: Academic Press Professional, 474-485 (1994).

[25] Scholkopf, B. and Smola, A., "Learning with kernels: Support vector machines, regularization, optimization and beyond, adaptive computation and machine learning," Cambridge, MA: The MIT Press (2002).

[26] Mery, D., Riffo, V., Zscherpel, U., Mondragn, G., Lillo, I., Zuccar, I., Lobel, H., and Carrasco, M., "Gdxray: The database of x-ray images for nondestructive testing," Journal of Nondestructive Evaluation 34.4, 1-12 (2015). 